S.C. Forth, ${ }^{1}$ D.J. Herman, ${ }^{\text {and M.A. James, }}$,

\title{
Fatigue Crack Growth Rate and Stress-Intensity Factor Corrections for Out-of-Plane Crack Growth
}

\begin{abstract}
REFERENCE: Forth, S. C., Herman, D. J., and James, M. A., "Fatigue Crack Growth Rate and Stress-Intensity Factor Corrections for Out-of-Plane Crack Growth," Fatigue and Fracture Mechanics: $34^{\text {th }}$ Volume, ASTM STP 1461, S. R. Daniewicz, J. C. Newman, Jr., and K. H. Schwalbe, Eds., ASTM International, West Conshohocken, PA, 2004.

ABSTRACT: Fatigue crack growth rate testing is performed by automated data collection systems that assume straight crack growth in the plane of symmetry and use standard polynomial solutions to compute crack length and stress-intensity factors from compliance or potential drop measurements. Visual measurements used to correct the collected data typically include only the horizontal crack length, which for cracks that propagate out-of-plane, under-estimates the crack growth rates and over-estimates the stress-intensity factors. The authors have devised an approach for correcting both the crack growth rates and stress-intensity factors based on twodimensional mixed mode-I/II finite element analysis (FEA). The approach is used to correct out-of-plane data for 7050-T7451 and 2025-T6 aluminum alloys. Results indicate the correction process works well for high $\Delta \mathrm{K}$ levels but fails to capture the mixed-mode effects at $\Delta \mathrm{K}$ levels approaching threshold $\left(\mathrm{da} / \mathrm{dN} \sim 10^{-10}\right.$ meter/cycle).
\end{abstract}

KEYWORDS: fatigue crack growth, mixed-mode, stress-intensity factor, aluminum, out-ofplane.

\section{Introduction}

Experimental testing for baseline fatigue crack growth rate properties has traditionally been performed on laboratory coupons designed to promote mode-I crack growth, where cracking is perpendicular to the applied load. However, material microstructure, residual stresses and other factors can cause the crack to turn out-of-plane and propagate in a mixed-mode manner. The ASTM Standard Test Method for Fatigue Crack Growth Rates (E 647), the testing standard used to develop fatigue crack growth rate data, limits the out-of-plane crack growth to within 20 degrees of the specimen symmetry plane for any growth increment over one-tenth the specimen width to maintain a reasonable accuracy of the mode-I equations. However, in some circumstances significant numbers of specimens may be invalid, or invalid by a small amount, directly impacting the value of a test program. For example, during a recent testing effort at NASA Langley Research Center on aluminum alloy 2025-T6 forgings, significant out-of-plane cracking was observed [1]. Nearly half of the test specimens had out-of-plane angles outside the ASTM E 647 limit of 20 degrees. One possible approach to recover some of this invalid data is to correct the crack growth rates and stress-intensity factors to account for crack

\footnotetext{
${ }^{1}$ Materials Research Engineer, NASA Langley Research Center, MS 188E, 2 W. Reid St., Hampton, VA 23681

${ }^{2}$ Student, Washington University in St. Louis, One Brookings Drive, St. Louis, MO 63130.

${ }^{3}$ Staff Scientist, National Institute for Aerospace, NASA Langley Research Center, MS 188E, 2 W. Reid St., Hampton, VA 23681
} 
turning. However, caution must be used when correcting data to avoid overlooking mixed-mode effects that can affect the data. The authors will present a methodology for correcting out-of-plane data and comment on the accuracy of modifying mixed-mode data.

\section{Mixed-Mode Crack Growth Data Correction}

A correction procedure can take more than one form, depending on the format in which the data is collected. For this study, the driving force and the crack growth rate will be considered separately because the experimental data was collected using a computer-controlled system based on compliance. To reduce measured compliance data into crack growth rate data, the compliance crack length is adjusted after the test is completed to match visual measurements taken periodically throughout the test. These visual measurements are taken along the symmetry plane of the specimen and represent the projected crack length, $\Delta \mathrm{a}$, defined in FIG 1. This procedure for automated data collection is described in detail within ASTM E 647. To assess the effect of mixed-mode crack growth on compliance values and computed stress intensity factors, finite element analyses were performed for several out-of-plane crack configurations.

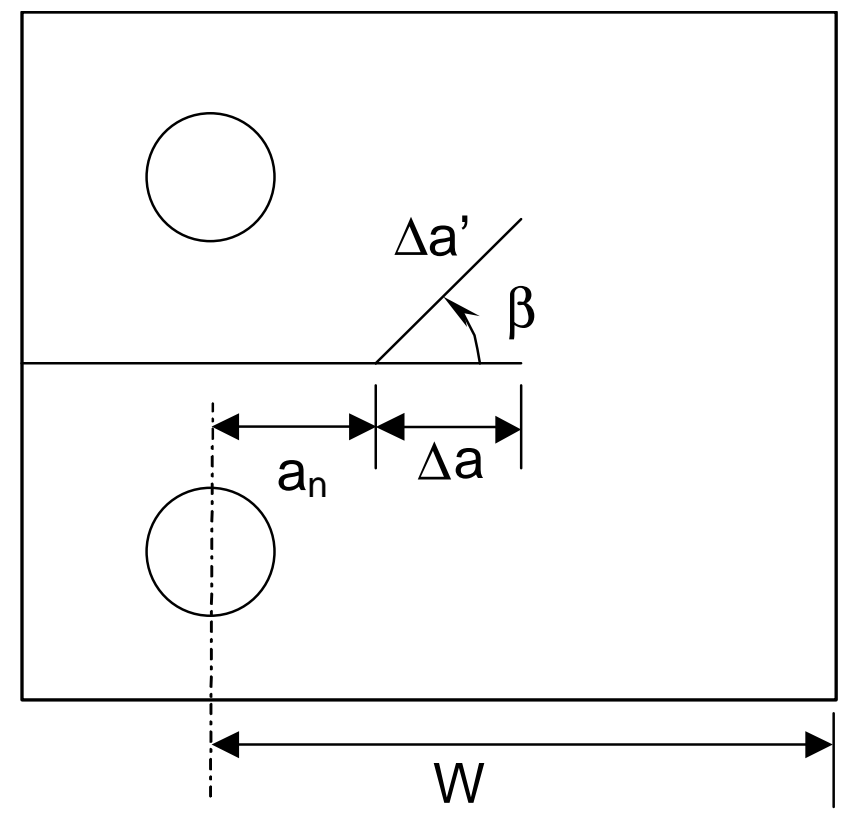

FIG 1-Out-of-plane crack growth configuration for a $C(T)$ specimen.

\section{Finite Element Analyses}

The finite element analysis (FEA) software FRANC2D/L [2, 3, 1] was used to calculate mode-I/II stress intensity factors (SIF's) for straight and angled crack configurations. A typical compact tension specimen, $\mathrm{C}(\mathrm{T})$, was considered with out-ofplane cracking. FIG 1 shows the configuration and nomenclature for the $\mathrm{C}(\mathrm{T})$ specimen studied herein, where the specimen dimensions for this study are: width $(\mathrm{W})=76.2 \mathrm{~mm}$, thickness $(B)=12.7 \mathrm{~mm}$, and notch length $\left(a_{n}\right)=19.05 \mathrm{~mm}$. The out-of-plane angle $\beta$ was varied from 0 degrees to 40 degrees. We assumed that the precrack and subsequent 
crack growth was in a straight line at an angle $\beta$ from the symmetry plane. In each case the projected crack length, $\Delta a$, was kept constant at $\Delta a=12.7 \mathrm{~mm}$ and the actual $\Delta a$ ' varied as

$$
\Delta a^{\prime}=\Delta a / \cos (\beta)
$$

The finite element analysis was used to determine straight-crack and mixed-mode stress-intensity factors (SIF). FIG. 2 shows mixed-mode $\mathrm{K}_{\mathrm{I}} \mathrm{SIFs}$ normalized by the straight-crack $(\beta=0)$ SIFs as open diamond symbols. As the out-of-plane angle $(\beta)$ increases, the mixed-mode $\mathrm{K}_{\mathrm{I}}$ value deviates from the straight-crack SIF, but for $\beta$ values less than 30 degrees the variation in SIF is less than one percent. Next, an energy release rate concept can be used to define a "mixed-mode total $\mathrm{K}$ " that is calculated as

$$
K_{T}=\sqrt{K_{I}^{2}+\gamma \cdot K_{I I}^{2}}
$$

where $\gamma=0.4$ for aluminum based on a curve fit to experimental data [5,6]. The open squares in FIG. 2 represent the mixed-mode total $\mathrm{K}$ and show a slighter higher dependence on angle than the mixed-mode $\mathrm{K}_{\mathrm{I}}$. This would be expected, as $\mathrm{K}_{\mathrm{II}}$ is increasing with out-of-plane angle. However, at an out-of-plane angle of 30-degrees the mixed-mode total $\mathrm{K}$ is still less than $1 \%$ greater than the straight-crack $\mathrm{K}_{\mathrm{I}}$.

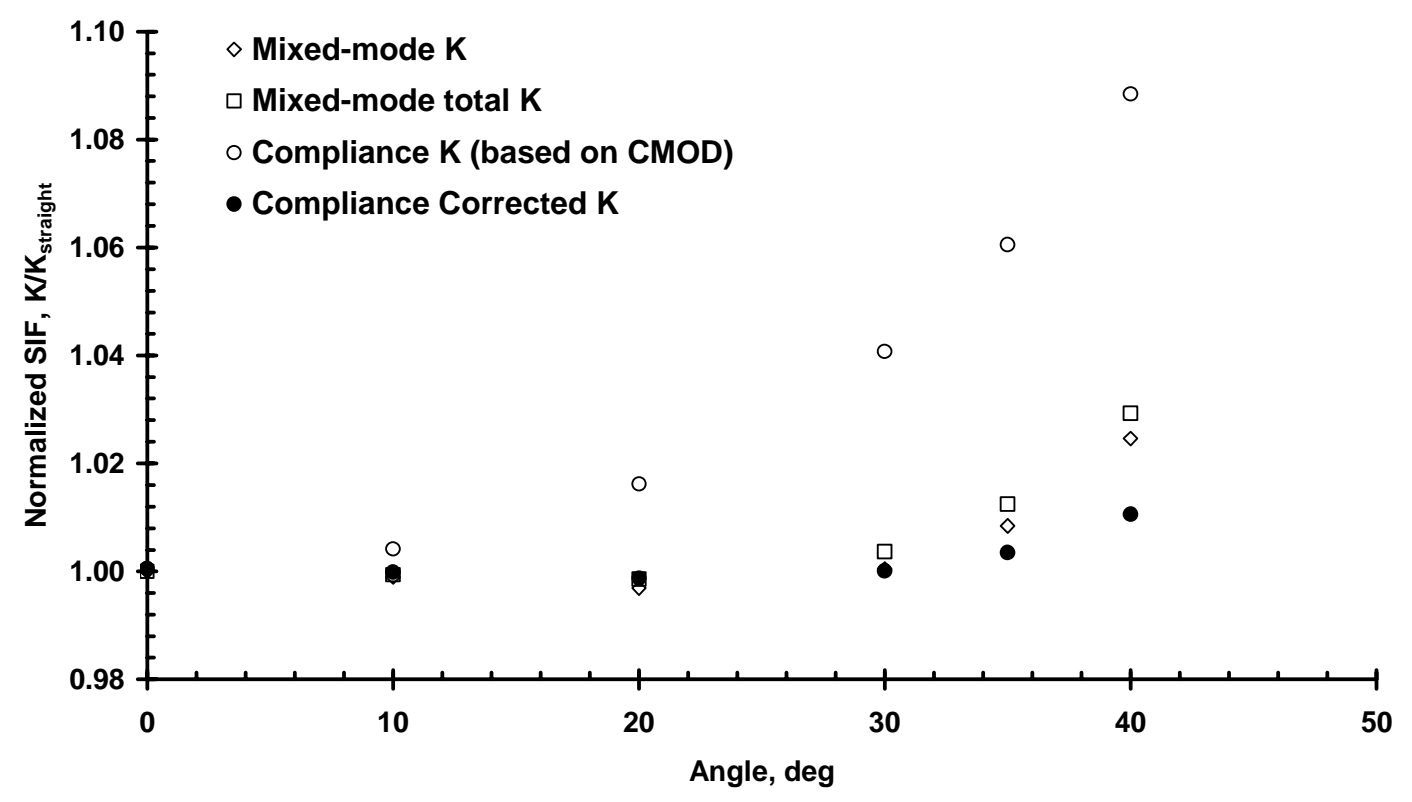

FIG. 2-Stress-intensity factor as a function of out-of-plane cracking angle.

Crack-mouth-opening displacements, CMOD, from the FEA results were also used to calculate the compliance crack length and stress intensity factor, SIF, values for each angle. The open circles in FIG. 2 show compliance K calculated from the analysis CMOD using the ASTM E 647 polynomial solutions for crack length and stress-intensity factor. The compliance solution for crack length significantly over-estimates the projected crack length and under-estimates the true crack length for out-of-plane cracking. (Actually, it does a reasonable job of estimating the vector length from the crack mouth to the crack tip, as one might expect.) The result of using CMOD for a mixed-mode crack is a poor estimate for the SIF during a test. In EIG. 2, SIF's calculated from the CMOD compliance crack length are noticeably in error outside $\beta=10$ degrees. 
The difference for 30 degrees is about 4\%. A simple solution is to correct the crack length polynomial equation such that it gives a corrected SIF. Note that this corrected crack length only has significance in that it represents an equivalent or effective crack length that gives the correct SIF. This may be thought of as part of the SIF calculation independent of the crack length (and growth rate) calculations.

The correction is simply to modify the polynomial equation for crack length (shorten the crack length):

$$
\Delta a=\Delta a_{c} \cdot \cos (f \beta)
$$

where $\Delta a_{c}$ is the compliance crack length from the polynomial equation and $f$ is a fraction of the out-of-plane cracking angle. One approach is to select the optimum angle fraction that minimizes the RMS error between the compliance K calculated from $\Delta a$ and the mixed-mode total $\mathrm{K}$. The difference in SIF between $f=0.5$ and the optimum is less than $1 \%$, so $f=0.5$ is used in all subsequent examples.

Using the above described correction, the filled circles in FIG. 2 show the "Compliance corrected K". The corrected values were in excellent agreement with the mixed-mode values for out-of-plane angles to 30 degrees. Even for angles of 35 and 40 degrees the agreement is within $3 \%$. But the simple approach taken here tends to overcorrect the data at 35 and 40 degrees.

\section{Correction Procedure}

For this study, the experiments were performed using an automated data acquisition system employing crack-mouth opening displacements (CMOD) to determine crack length. The CMOD values along with feedback from the servo-hydraulic test machine were input into a computer system that automatically controlled the test using the guidelines set forth in ASTM E 647. The form of data acquisition used dictated the outof-plane correction procedure incorporated herein.

The testing was performed under K-control, i.e. load is continually adjusted based upon crack length estimated from compliance. Visual measurements of the crack length measured horizontally along the specimen symmetry plane, $\Delta a$, were taken periodically throughout the test. The compliance crack length is then adjusted to fit the visual measurements. Crack growth rates are then corrected by dividing the projected crack length $\Delta a$, by the cosine $(\beta)$. This will adjust the rates to better account for the actual path traveled by the crack, $\Delta a$ '. For out-of-plane angles less than 30 degrees, the stress intensity factors, SIFs, are not corrected because the compliance data has already been reduced using the projected crack lengths. As shown in FIG. 2, using the projected crack length to compute the SIFs yields reasonable accuracy compared to the mixed-mode $\mathrm{K}_{\mathrm{I}}$. For out-of-plane angles exceeding 30 degrees, the "compliance corrected K" procedure described above must be used.

\section{0-T7451 Mixed-Mode Data}

Donald [7] performed tests on 7050-T7451 in the S-L orientation with varying crack inclination angles relative to the rolling direction. This approach promoted crack growth out-of-plane in the direction of the inclined rolling direction. Results are presented for cracking angles of 1, 10, 17 and 26 degrees as shown in FIG. 3. Tests were performed at a stress ratio $(\mathrm{R})$ of 0.7 and at a crack growth rate above $10^{-9}$ meters/cycle. The results 
support the ASTM E 647 guidelines for out-of-plane cracking showing that crack growth rates are affected by out-of-plane angle.

Donald reported the applied $\Delta \mathrm{K}$ and the projected crack length $(\Delta \mathrm{a})$, as would be expected from visual measurements. The FEA results presented in FIG. 2 show that for $\mathrm{K}$ control based on compliance crack length, the applied $\mathrm{K}$ can differ significantly from the actual $\mathrm{K}$ at the tip. To apply a correction to the data, the compliance crack length must be known. By means of equation (3), an estimate of the compliance crack length can be computed from the projected crack length. Using the projected crack length, we can estimate the applied load for a given $\Delta \mathrm{K}$, and calculate the correct crack length and subsequently a corrected $\Delta \mathrm{K}$. The growth rate is then corrected by dividing the projected crack length growth rate by the cosine of the out-of-plane angle $\beta$.

Donald also provided baseline fatigue crack growth rate data over a range of $\Delta K$ values from about 2 to $5 \mathrm{MPa} \mathrm{m}{ }^{1 / 2}$. FIG. 3 compares the average values of growth rate for uncorrected and corrected data sets with the baseline data. The uppermost closed triangle symbol shows the average for the 1 degree specimens. This data point is in excellent agreement with the baseline data and is not corrected. The remaining closed triangles show average values for the uncorrected data. As the out-of-plane angle increases, the growth rates deviate more from the baseline data. The closed squares show average values for the corrected data. The corrected values agree very well with the baseline data. These data show the importance of correcting both the growth rates and the driving force. If only the growth rates were corrected, the agreement would not be nearly as good. FIGS 4 and 5 show the full data set for the 17 and 26 degree cases respectively. The open circles show the baseline data and the closed triangles show the uncorrected out-of-plane data. The open squares show the corrected data. The agreement is excellent.

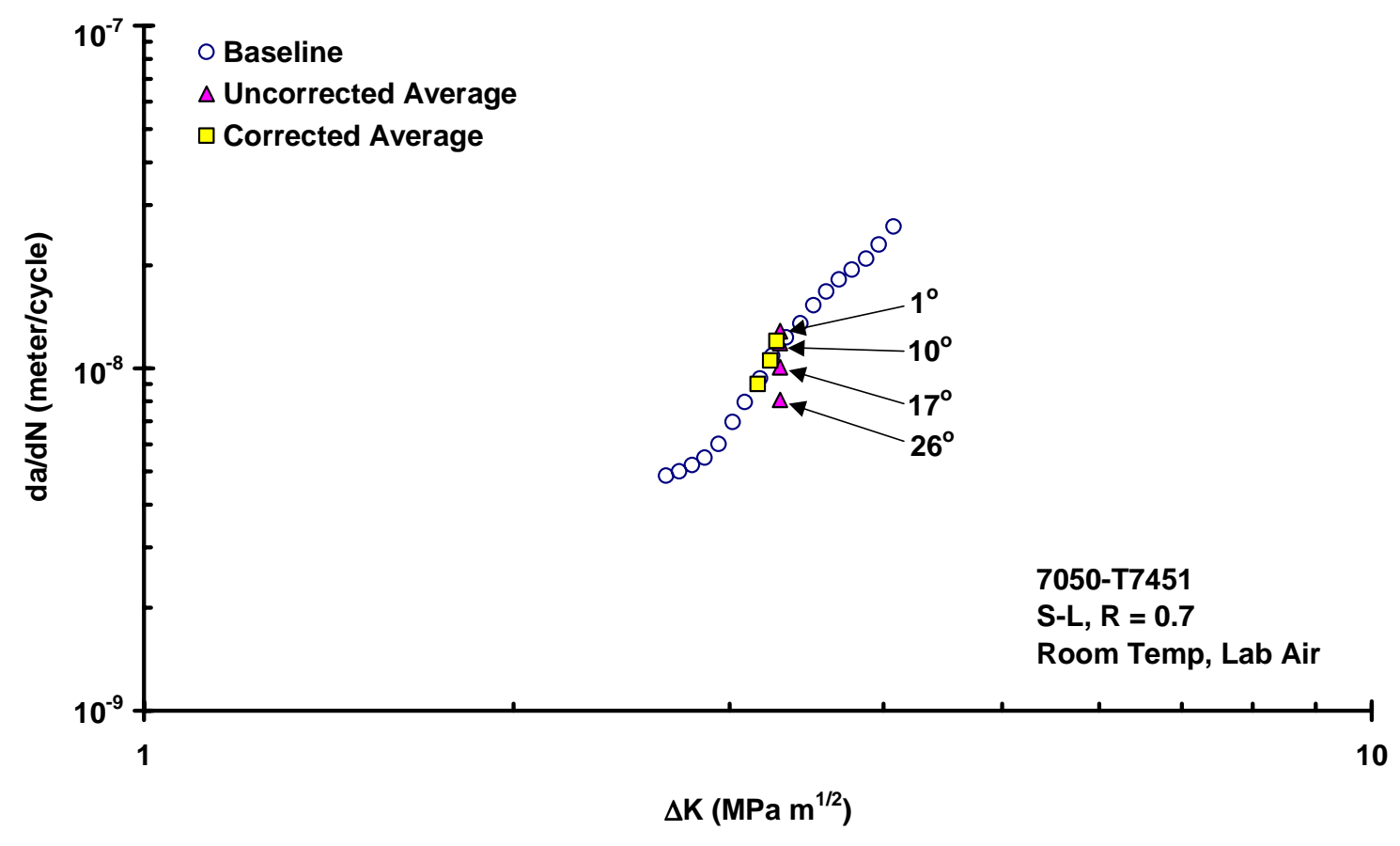

FIG. 3 - Comparison of corrected data with original and baseline data. 


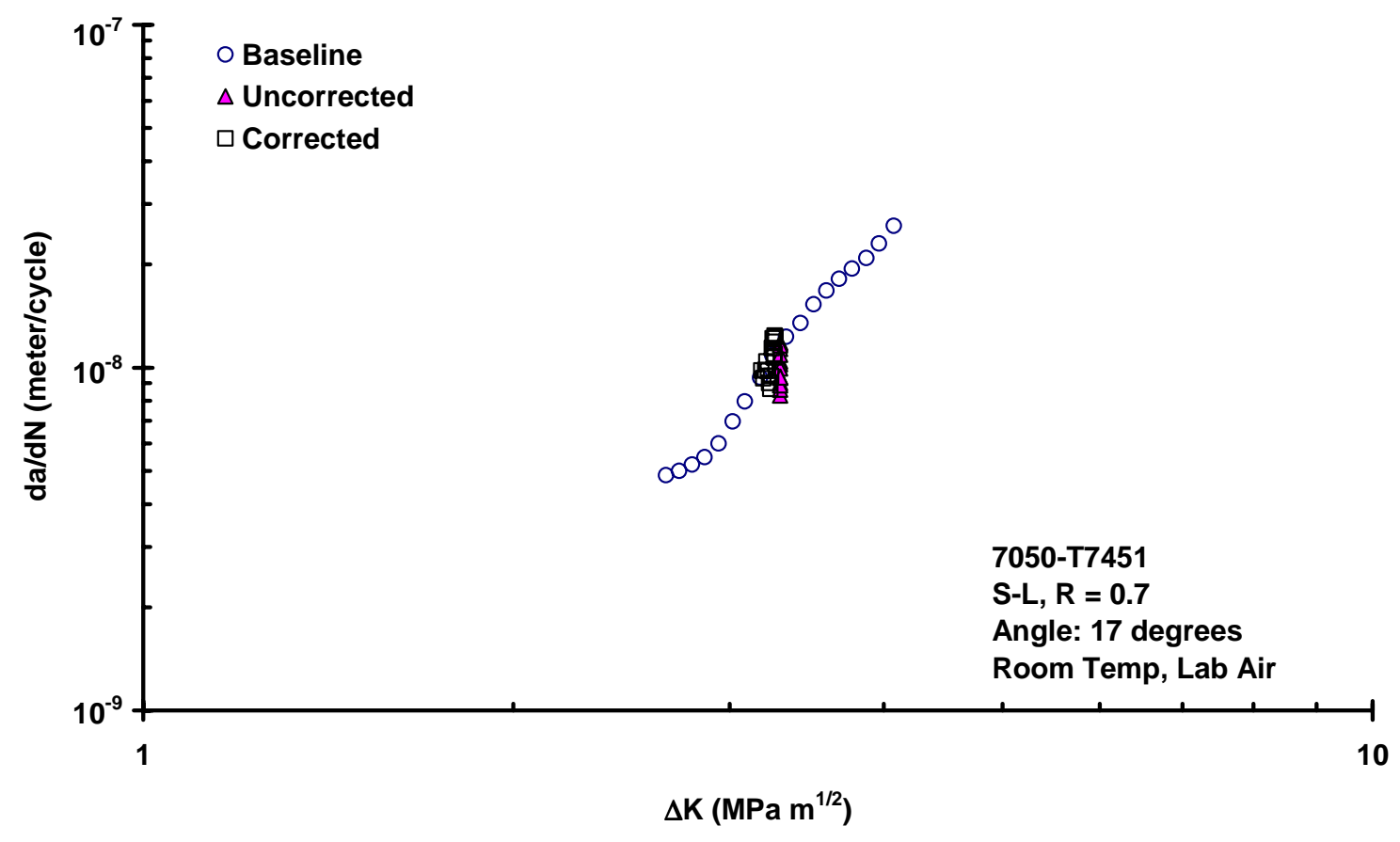

FIG. 4-Comparison of corrected data with original and baseline data for the 17 degree case.

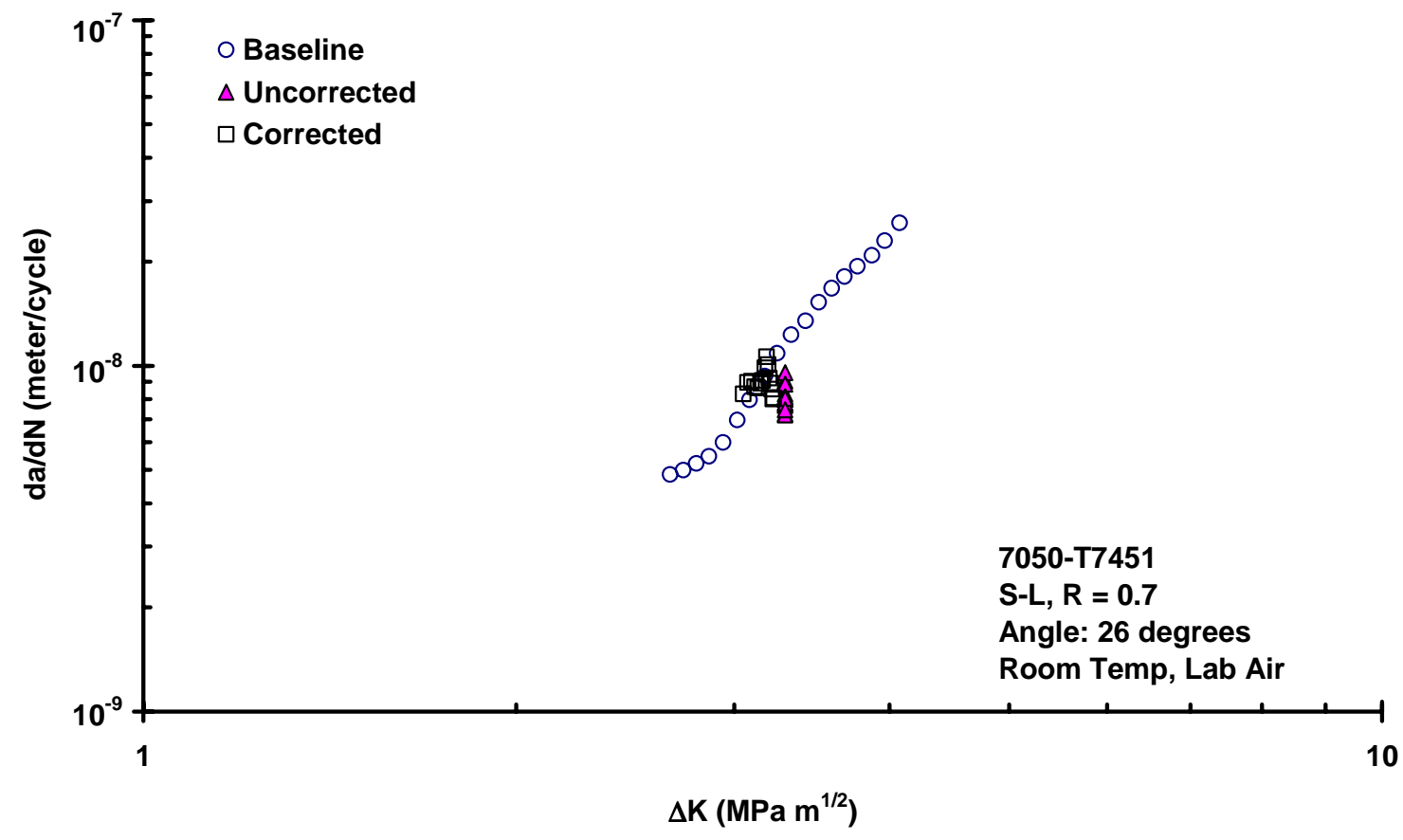

FIG. 5-Comparison of corrected data with original and baseline data for the 26 degree case.

\section{5-T6 Mixed-Mode Data}

Test specimens were machined from forged aluminum alloy 2025-T6 propeller spars that were provided by a propeller manufacturer. These spars were provided in a near-netshape form, so a finished propeller requires little additional machining. Each propeller 
spar is forged from uniform material stock, e.g., cylindrical billets, so the material is substantially deformed during the forging process. The mechanical work of the forging process resulted in weak microstructural planes that promoted out-of-plane cracking. More information can be found in reference Forth, et. al. [1]. EIG. 6 shows the average out-of-plane angle for each of the specimens tested, and the out-of-plane angles are presented in Table 1. For the 60 specimens, there were 16 straight cracks and 24 tests outside the ASTM E 647 limit of 20 degrees. The remaining specimens were not straight, but were within the 20 degree limit for crack path straightness.

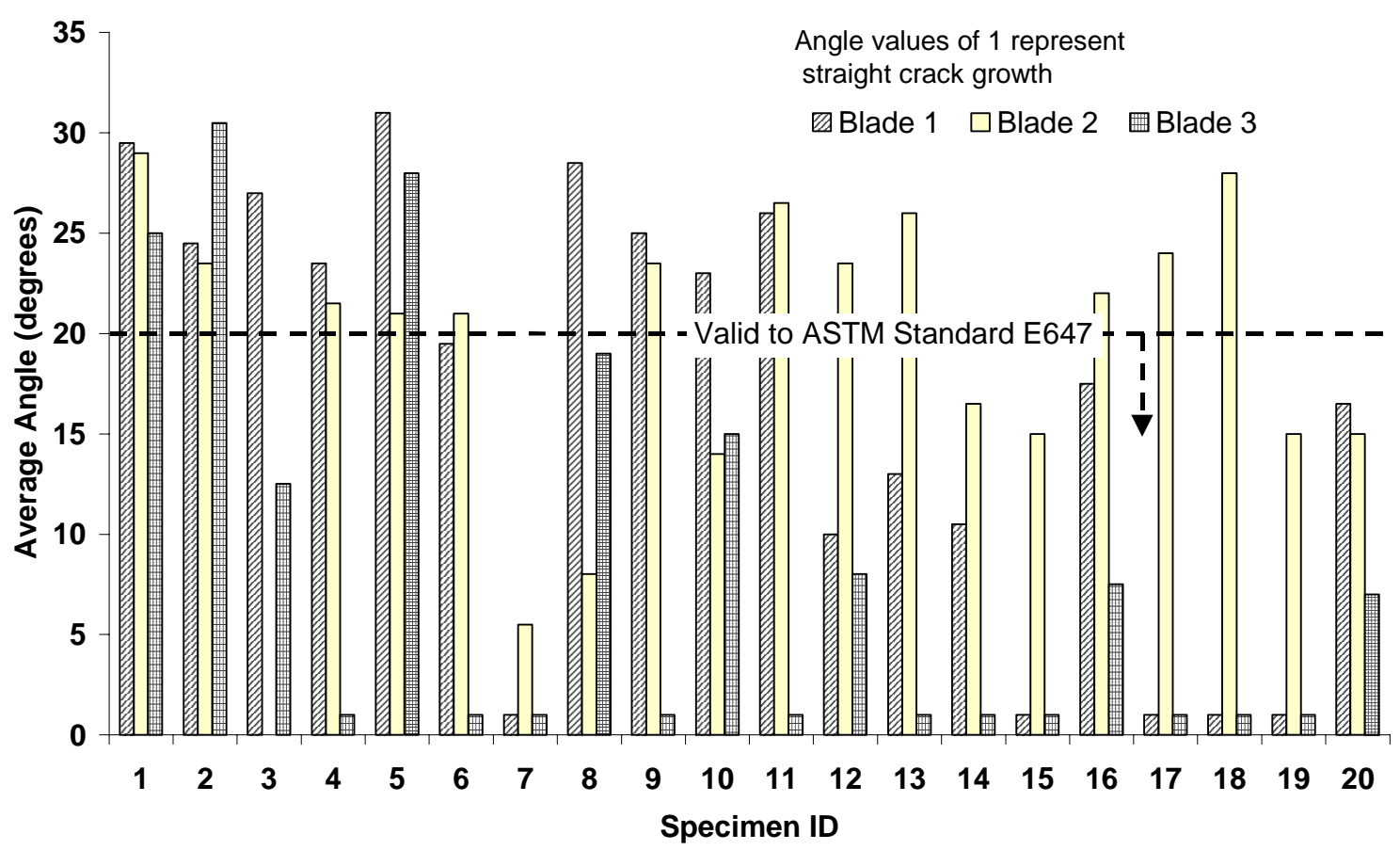

FIG. 6-Average out-of-plane angle from centerline for fatigue crack growth tests.

TABLE 1-Out-of-plane angle (front/back) from centerline for fatigue crack growth

\begin{tabular}{c|c|c|c}
\hline Specimen ID & $\begin{array}{c}\text { tests } \\
\text { Out-of-plane angle } \\
\text { (front/back) }\end{array}$ & $\begin{array}{c}\text { Blade 2 } \\
\text { Out-of-plane angle } \\
\text { (front/back) }\end{array}$ & $\begin{array}{c}\text { Blade 3 } \\
\text { Out-of-plane angle } \\
\text { (front/back) }\end{array}$ \\
\hline 1 & $31 / 28$ & $26 / 32$ & $25 / 25$ \\
2 & $27 / 22$ & $25 / 22$ & $31 / 30$ \\
3 & $25 / 29$ & untested & $10 / 15$ \\
4 & $25 / 22$ & $21 / 22$ & $0 / 0$ \\
5 & $29 / 33$ & $18 / 24$ & $30 / 26$ \\
6 & $22 / 17$ & $17 / 37$ & $0 / 0$ \\
7 & $0 / 0$ & $0 / 11$ & $0 / 0$ \\
8 & $30 / 27$ & $8 / 8$ & $16 / 22$ \\
9 & $28 / 22$ & $23 / 24$ & $0 / 0$ \\
10 & $23 / 23$ & $13 / 15$ & $13 / 17$ \\
11 & $22 / 30$ & $30 / 23$ & $0 / 0$ \\
12 & $12 / 8$ & $29 / 18$ & $9 / 7$ \\
13 & $17 / 9$ & $26 / 26$ & $0 / 0$ \\
14 & $11 / 10$ & $19 / 14$ & $0 / 0$ \\
15 & $0 / 0$ & $15 / 15$ & $0 / 0$ \\
\hline
\end{tabular}




\begin{tabular}{c|c|c|c}
\hline Specimen ID & $\begin{array}{c}\text { Blade 1 } \\
\text { Out-of-plane angle } \\
\text { (front/back) }\end{array}$ & $\begin{array}{c}\text { Blade 2 } \\
\text { Out-of-plane angle } \\
\text { (front/back) }\end{array}$ & $\begin{array}{c}\text { Blade 3 } \\
\text { Out-of-plane angle } \\
\text { (front/back) }\end{array}$ \\
\hline 16 & $16 / 19$ & $24 / 20$ & $7 / 8$ \\
17 & $0 / 0$ & $28 / 20$ & $0 / 0$ \\
18 & $0 / 0$ & $28 / 28$ & $0 / 0$ \\
19 & $0 / 0$ & $12 / 18$ & $0 / 0$ \\
20 & $9 / 24$ & $12 / 18$ & $12 / 2$ \\
\hline
\end{tabular}

Fatigue crack growth rate data was generated using fixed stress ratios of 0.05, 0.1 and 0.7 and using constant $\mathrm{K}_{\max }$ values of $11,13.7,16.4,22$ and $33 \mathrm{MPa} \mathrm{m}^{1 / 2}$ per ASTM E 647. The specimen test data presented is grouped and plotted based on high and low stress ratio in FIG. 7 and FIG. 8 respectively. Specimens presented in these plots were tested using the constant $\mathrm{R}$ and $\mathrm{K}_{\max }$ load reduction methods to determine threshold, defined as $\mathrm{da} / \mathrm{dN} \sim 10^{-10}$ meter/cycle, and using the constant $\mathrm{R}$ load increasing method to determine the upper portion of the crack growth rate curve, as indicated by the figure legends. The constant R load reduction and load increasing tests are denoted with "LR" and "LI", respectively. The specimen number is denoted in the figure legend to correlate test data to out-of-plane angle. The majority of the constant $\mathrm{R}$, load increasing tests were performed following the constant $\mathrm{K}_{\max }$ and $\mathrm{R}$ load reduction tests, resulting in duplicate specimen numbers in the figure legends.

All of the out-of-plane data was corrected using the previously described procedure. An example of the effect that the correction procedure has on the data is presented in FIG. 9. Specimen number 4 from blade number 1 (4-b1) was chosen for examination because the average out-of-plane angle was approximately 23-degrees. The original data obtained during the test is labeled "compliance." The data was then adjusted for $\Delta \mathrm{K}$ using visual measurements along the symmetry plane, labeled "adjusted K." Finally, the fatigue crack growth rate was corrected to the actual crack length and this data set is labeled "fully corrected." The correction procedure yielded a small shift in the data, as would be expected with an out-of-plane angle of less than 30 degrees based on the preceding analyses. 


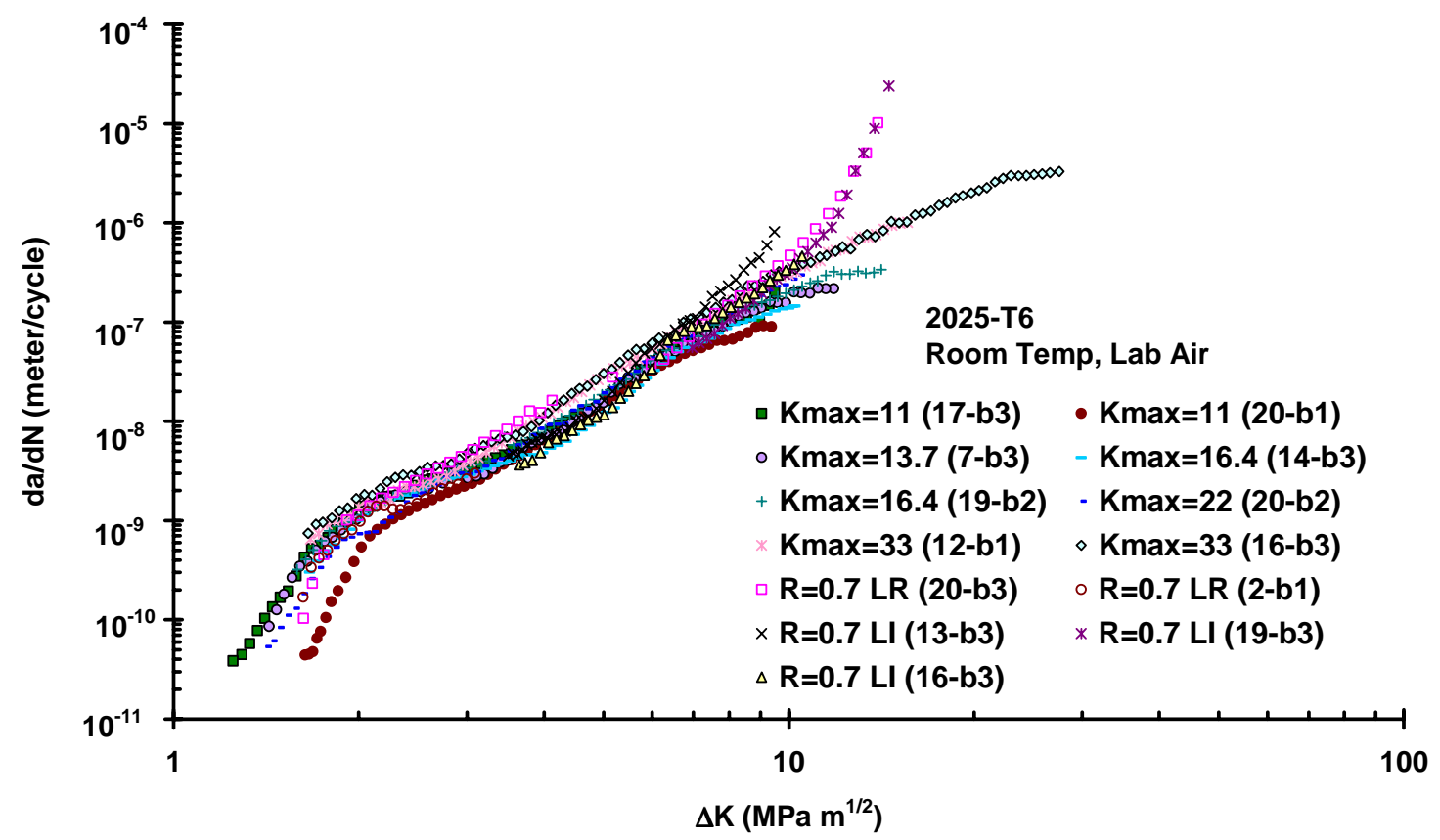

FIG. 7- High stress ratio fatigue crack growth rate data for aluminum alloy 2025-T6.

(All data is corrected for out-of-plane angle.)

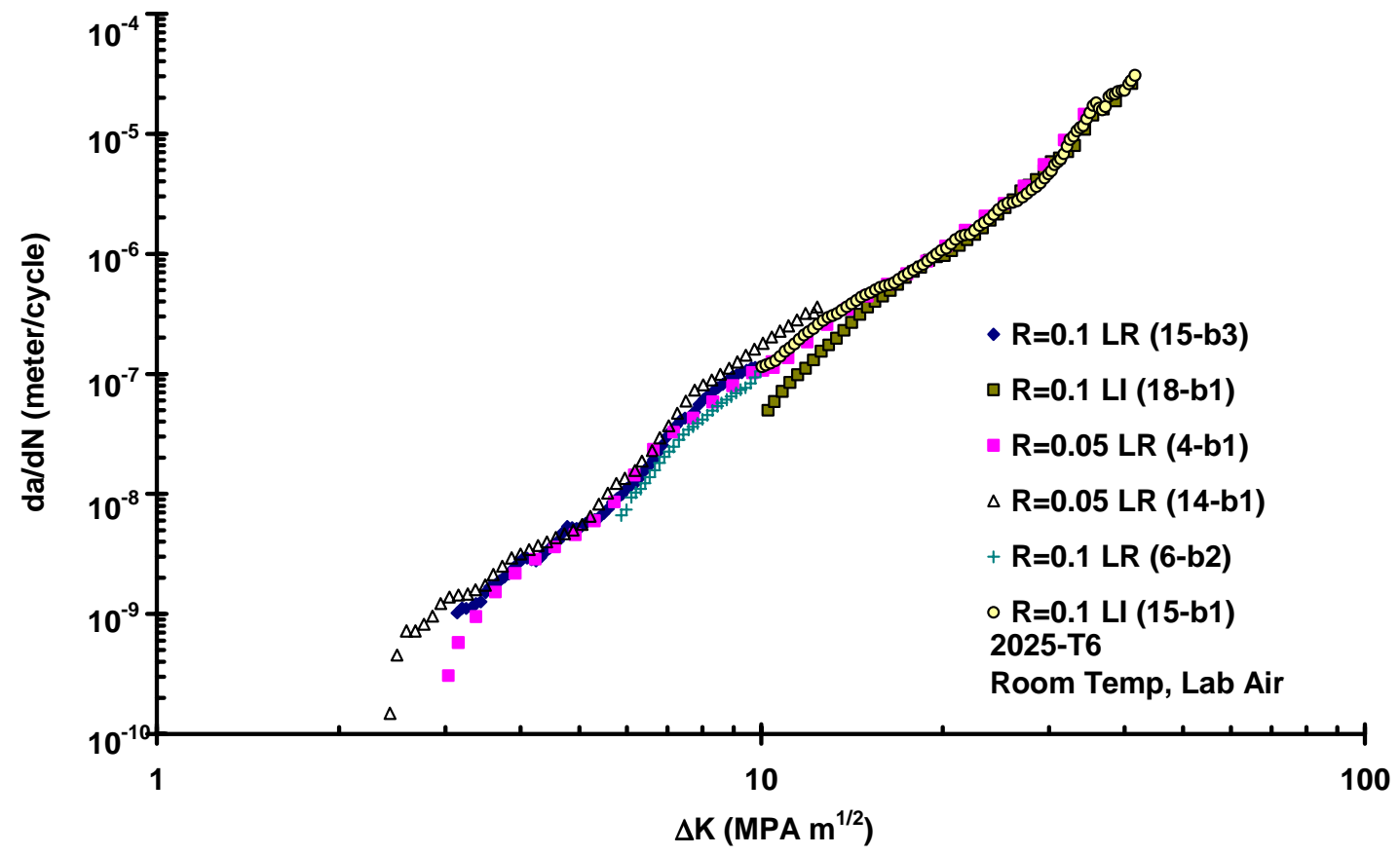

FIG. 8- Low stress ratio fatigue-crack-growth-rate data for alloy 2025-T6. (All data is corrected for out-of-plane angle.) 


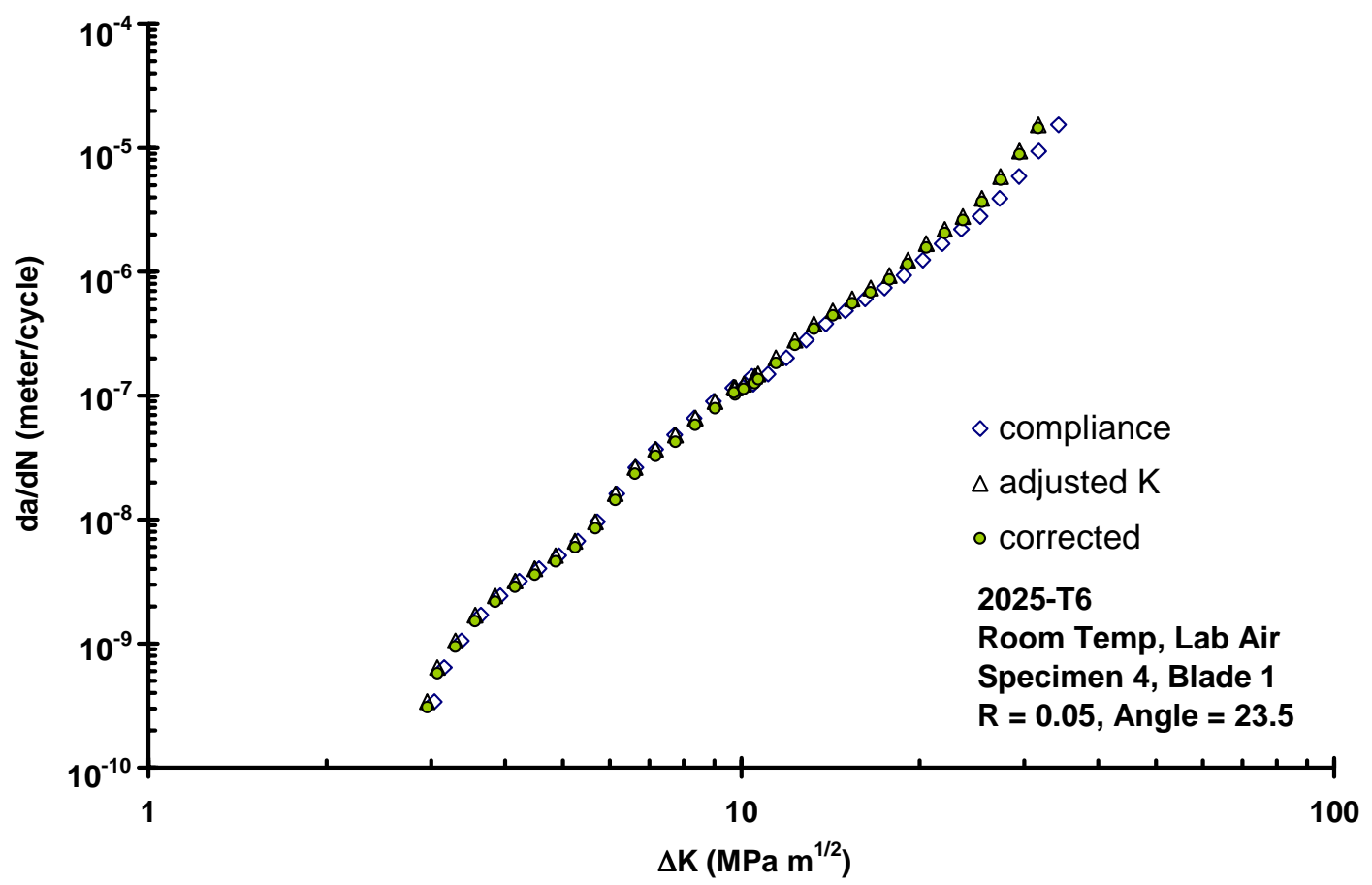

FIG. 9-Effect of corrections on the fatigue crack growth rate data of specimen 4, blade $1(R=0.05 L R)$.

\section{Effect of Mode-Mixity on Crack Growth Rate}

Donald 17 generated data in 7050-T7451 at a stress ratio of 0.7 and a crack growth rate above $10^{-9}$ meters/cycle. The combination of high stress ratio and relatively high $\Delta \mathrm{K}$ minimizes energy dissipation under mixed-mode loading due to roughness or other mechanisms that may influence growth rates in mixed-mode [8]. The 2025-T6 data was generated at both high and low stress ratios and over a wide range of crack growth rates. To investigate the effect mode-mixity is having on fatigue crack growth, both the high and low stress ratio data are investigated. The high $\mathrm{R}$ data will isolate the ranges of crack growth rate that are affected without the inclusion of closure due to load ratio [9]. The low R data will likely be more influenced by plasticity- or roughness-induced closure, or other mechanisms that can reduce the driving force in mixed-mode situations [9].

To investigate the high stress ratio data, constant $\mathrm{K}_{\max }$ and constant $\mathrm{R}=0.7$ load reduction test specimens were chosen. The constant $\mathrm{K}_{\max }$ test should minimize the influence of plasticity- and roughness-induced crack closure [10]. Therefore, discrepancies between the tests may be attributable to mixed-mode phenomenon. The crack growth rate versus stress intensity for the constant $K_{\max }$ tests are plotted in FIG. 10. Comparing the constant $\mathrm{K}_{\max }=11 \mathrm{MPa} \mathrm{m}{ }^{1 / 2}$ tests, specimen 17-b3 propagated straight whereas specimen 20-b1 propagated an average of 16.5 degrees out-of-plane (front/back $=9 / 24)$. At higher $\Delta \mathrm{K}$ levels $\left(\Delta \mathrm{K}>5 \mathrm{MPa} \mathrm{m}{ }^{1 / 2}\right)$, there is little difference in the tests. However, as the $\Delta \mathrm{K}$ reduces below $4 \mathrm{MPa} \mathrm{m}^{1 / 2}$, the data sets diverge with the higher outof-plane angle having lower crack growth rates. Further comparison of specimen 20-b1 to specimens 19-b2 and 20-b2 illustrates the effect of twisting (front-to-back out-of-plane angle variation) in the specimen, which could be indicative of mode-III behavior. Each 
test propagated out-of-plane at approximately the same average angle, however specimen 20-b1 had significant twisting. Near threshold, the twisting exhibited in specimen 20-b1 translated into an order of magnitude decrease in the crack growth rate, whereas specimens 19-b2 nearly matched the straight data. Specimen 20-b2 fell between the other data and suggests that the out-of-plane angle may influence the crack growth rate more than specimen 19-b2 suggests.

The constant $\mathrm{R}=0.7$ data is presented in FIG. 11 as crack growth rate versus stress intensity. Specimen 2-b1 propagated an average of 24.5 degrees out-of-plane. Unfortunately, there is no overlap of specimen 2-b1 data with the straight tests. However, extrapolating the data from specimen 2-b1, it would appear to have the same crack growth rates at $\Delta \mathrm{K}$ values exceeding $5 \mathrm{MPa} \mathrm{m}^{1 / 2}$, similar to the constant $\mathrm{K}_{\max }$ data presented in FIG. 10. Specimen 20-b3 exhibited significant twisting, much like specimen 20-b1 discussed previously, and does overlap the straight data. Once again, the crack growth rates from specimen 20-b3 do not coincide with the straight data until $\Delta \mathrm{K}$ exceeds $5 \mathrm{MPa} \mathrm{m}^{1 / 2}$. A comparison of specimens $20-\mathrm{b} 3$ and 2-b1 near threshold $(\Delta \mathrm{K}<2 \mathrm{MPa}$ $\mathrm{m}^{1 / 2}$ ) show similar crack growth behavior. It appears the effect of the high out-of-plane angle of specimen 2-b1 and the twisting of specimen 20-b3 have coincidentally generated the same crack growth rates.

The low stress ratio data is presented in FIG. 12 for two tests performed using the constant $\mathrm{R}=0.05$ load reduction method. The data is presented for average out-of-plane angles of 10.5 and 23.5 degrees from specimens 14-b1 and 4-b1 respectively. The low stress ratio data exhibits the same trends as the high stress ratio data. The high $\Delta \mathrm{K}$ data does not appear to be dependent on out-of-plane angle, whereas the low stress data, $\Delta \mathrm{K}<$ $5 \mathrm{MPa} \mathrm{m}{ }^{1 / 2}$, diverges. Once again, the higher out-of-plane angle corresponds to lower growth rates at the same $\Delta K$. However, both specimens were tested from a high initial $\Delta \mathrm{K}$, which could exacerbate any closure phenomenon leading to a high threshold. Neither of these specimens exhibited significant twisting. 


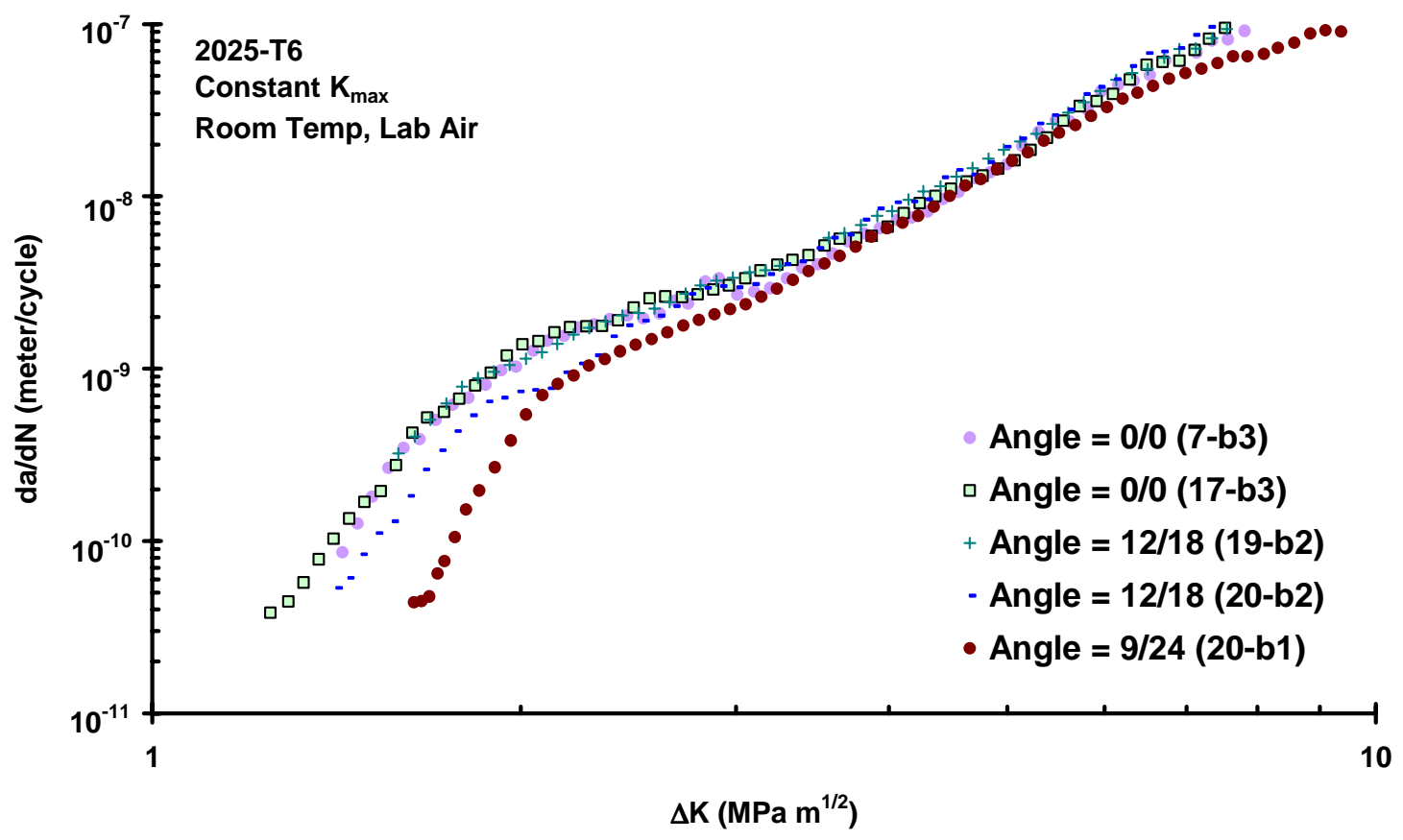

FIG. 10. Constant $K_{\max }$ data near threshold for different out-of-plane angles. (All data is corrected for out-of-plane angle.)

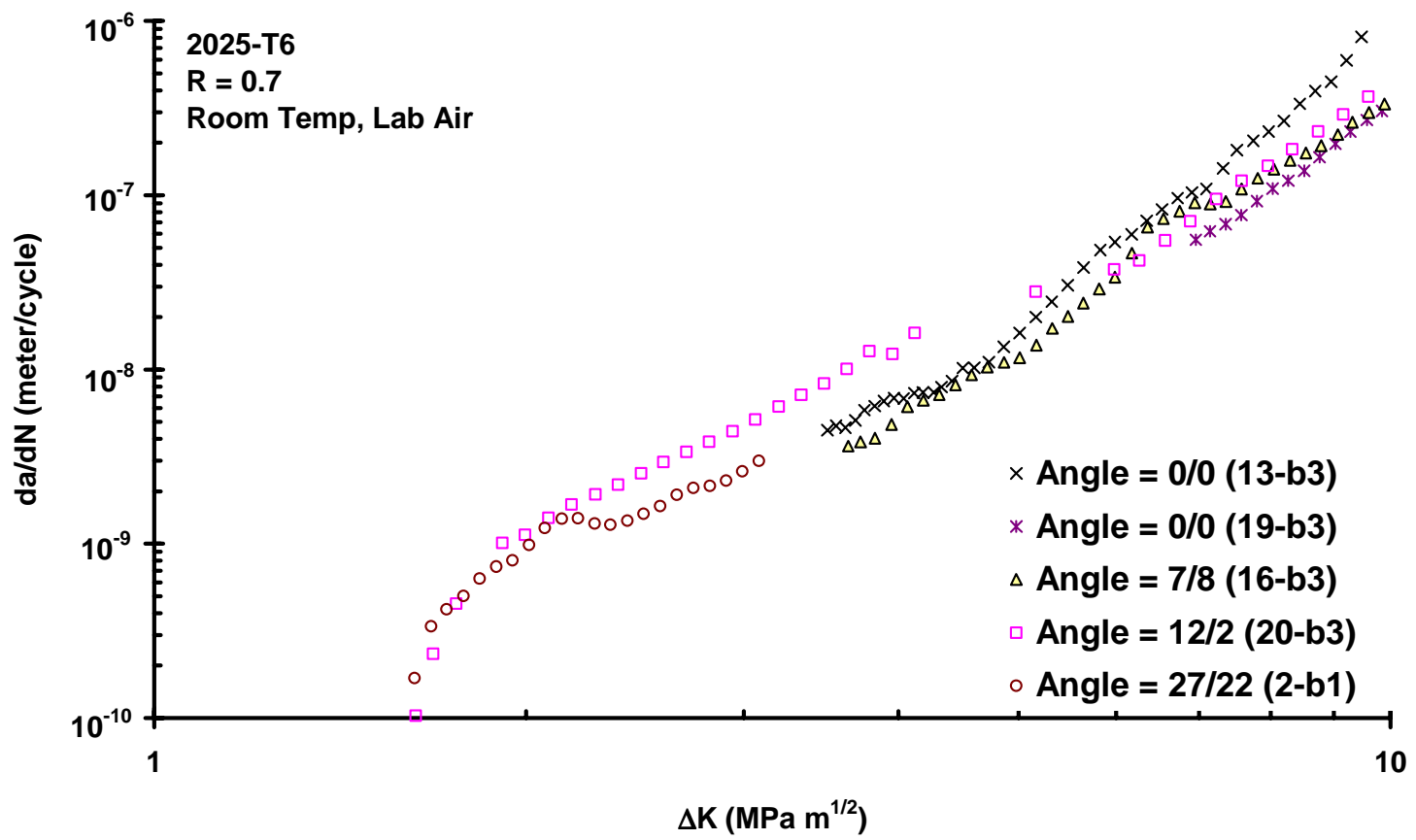

FIG. 11. Constant $R=0.7$ data near threshold for different out-of-plane angles. (All data is corrected for out-of-plane angle.) 


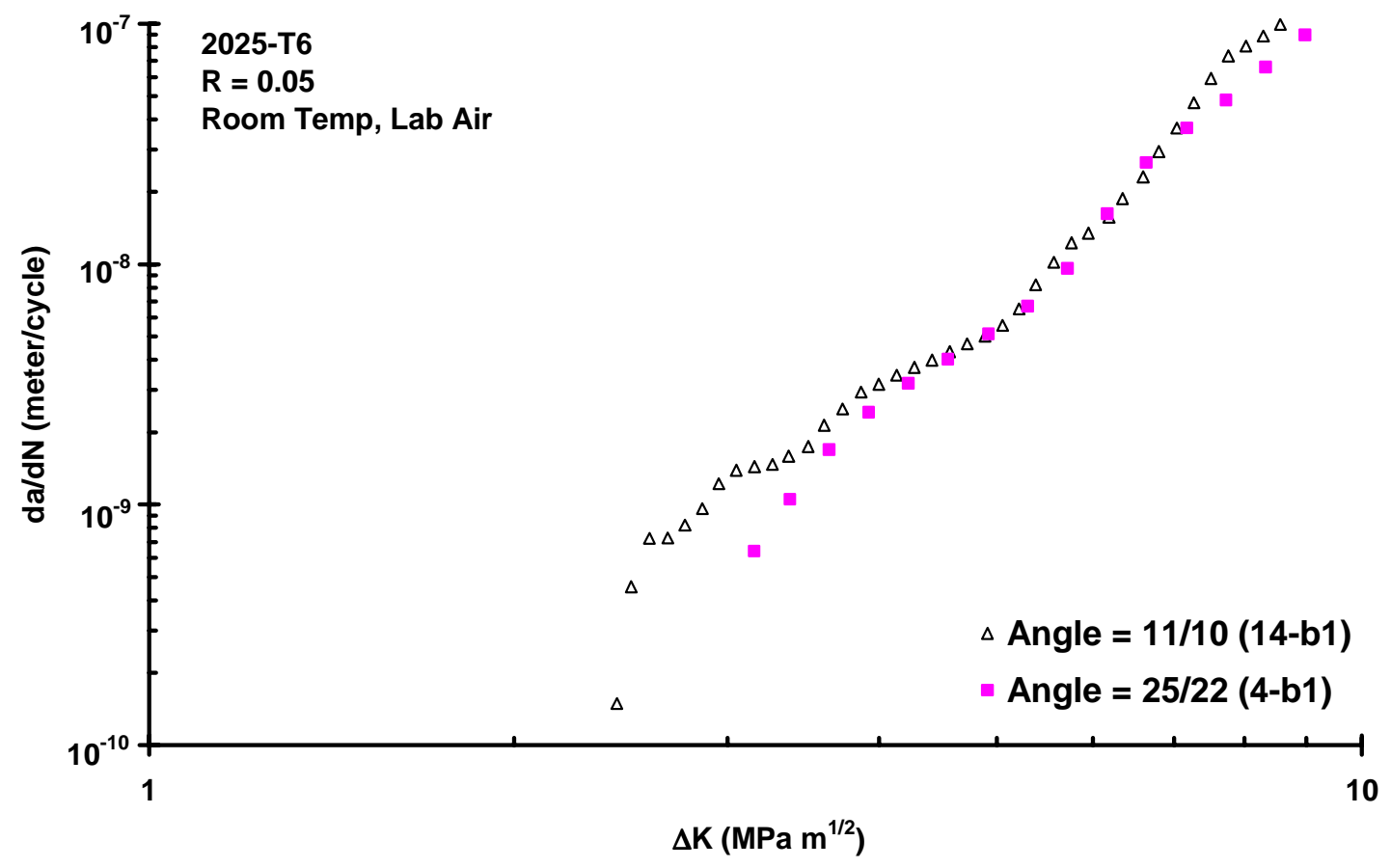

FIG. 12. Constant $R=0.05$ data near threshold for different out-of-plane angles. (All data is corrected for out-of-plane angle.)

\section{Discussion}

The authors have presented a very simple procedure for correcting out-of-plane crack growth data within 40 degrees. The correction procedure was developed using mixedmode finite element analyses modeling the compact tension specimen, $\mathrm{C}(\mathrm{T})$. The result of these analyses was that both the crack growth rate and stress intensity solution must be corrected to account for the out-of-plane cracking. The crack growth rate can be corrected by either adjusting the compliance polynomial solution defined in ASTM E 647 or the projected crack length measured visually on the specimen surface. The stress intensity factors can then be corrected using the projected crack length in the calculations.

When out-of-plane cracking occurs, the polynomial equations defined in ASTM E 647 under-estimate the actual crack length and over-estimate the projected crack length. Growth rates are also under-estimated because crack length measurements are typically made only of the projected crack length, rather than the true path. These high-level continuum descriptions are only simple approximations to the true behavior along a crack front, where the crack path is not straight, but locally influenced by microstructure, and the local stress intensity factor (SIF) is only a convenient approximation to the cyclic deformations that drive the growth. When compared to a "straight" crack, the out of plane crack likely has a more tortuous path, and the local SIF's are consequently influenced both by the global mixed-mode behavior as well as the local crack path deviations. In addition, out-of-plane cracking likely has larger asperities on the crack plane, and those asperities may contribute to reduced growth rates by dissipating energy as friction.

The correction procedure was initially validated using 7050-T7451 aluminum alloy data available in the literature [7]. The out-of-plane cracking was encouraged by machining specimens with the S-L material axis rotated with respect to the crack 
symmetry plane of the $\mathrm{C}(\mathrm{T})$ specimen. As a result, in each case the cracking was essentially in the S-L material plane resulting in a relatively smooth and consistent fracture surface. Further, the data was generated at a high stress ratio and $\Delta \mathrm{K}$ level to minimize crack face interaction effects. The correction procedure worked well in collapsing the out-of-plane data with the "straight" data.

The correction procedure was also applied to 2025-T6 aluminum alloy. The out-ofplane data was generated in this material from a weak microstructural plane being established in the forging process. The correction procedure was applied to this data at both high and low stress ratios with some success. However, if the average out-of-plane angles exceeded 15 degrees the data could not be reliably corrected near threshold, da/dN $\sim 10^{-10}$ meter/cycle because mixed-mode effects, such as roughness-induced closure, became dominant. Furthermore, specimens that displayed significant twisting, i.e. the difference in out-of-plane angle measured on the specimen front and back exceeded 10 degrees, could not be reliably corrected near threshold, da/dN $\sim 10^{-10}$ meter/cycle. It is believed that the mixed-mode behavior at the threshold $\Delta \mathrm{K}$ level is dominant and a simple correction procedure is inadequate.

\section{Conclusion}

In conclusion, a simple procedure has been developed to correct out-of-plane data to account for mixed-mode effects. Application of this procedure to test programs that experience significant, unexpected out-of-plane cracking may aid in generating useable data. However, this procedure cannot be reliably used for crack growth rates approaching the fatigue crack growth threshold (da/dN $\sim 10^{-10}$ meter/cycle) when the outof-plane angle exceeds 15 degrees. Furthermore, this procedure is invalid for correcting data that has significant variation in the front-to-back out-of-plane angle, i.e. twisting or mode-III type behavior. The mixed-mode phenomenon of both these cases is beyond the scope of a simple continuum-based approach to salvage out of specification data.

\section{References}

[1] Forth, S. C., James, M. A., Newman, J. A., Everett, R. A. and Johnston, W. M., "Mechanical Properties of 2025-T6 Aluminum," NASA Technical Memorandum, 2004.

[2] James, M. and Swenson, D., "FRANC2D/L: A Crack Propagation Simulator for Plane Layered Structures," Online, Available: http://www.mne.ksu.edu/ franc2d/, 2002.

[3] Wawrzynek, P. A., and Ingraffea, A. R., "Integrated finite element analysis of fracture processes: an integrated approach," Theoretical and Applied Mechanics, No. 8, 1987, pp. 137-150.

[4] M. A. James and D. V. Swenson, "A Software Framework for Two Dimensional Mixed Mode-I/II Elastic-Plastic Fracture," Mixed-Mode Crack Behavior, ASTM STP 1359, K. J. Miller and D. L. McDowell, Eds., American Society for Testing and Materials, 1999.

[5] Aoki, S., Kishimoto, T., Yoshida, T., Sakata, W., and Richard, H.A., J. Mech. Phys. Solids, Vol 38, 1990, pp $195-213$.

[6] Forth, S. C., Keat, W. D., and Favrow, L. H., "Experimental and computational investigation of three-dimensional mixed-mode fatigue," Fatigue and Fracture of Engineering Materials and Structures, Vol 25, 2002, pp 3-15.

[7] Donald, J. K., "The Effect of Out-of-Plane Cracking on FCGR Behavior," ASTM 
Research Report \#E8-1001, December 12, 1995.

[8] Lam, Y.C., "Mixed mode fatigue crack growth with a sudden change in loading direction," Theoretical and Applied Fracture Mechanics, Vol 19, 1993, pp 69-74.

[9] Newman, Jr., J.C. and Piascik, R.S. (Eds.), Fatigue Crack Growth Thresholds, Endurance Limits, and Design, ASTM STP 1372, American Society for Testing and Materials, West Conshohocken, PA, 2000.

[10] Smith, S.W. and Piascik, R.S., "An Indirect Technique for Determining ClosureFree Fatigue Crack Growth Behavior," Fatigue Crack Growth Thresholds,

Endurance Limits, and Design, ASTM STP 1372, Newman, Jr., J.C. and Piascik, R.S., Eds., American Society for Testing and Materials, 2000, pp. 109-122. 